\title{
TCP-Swift: An End-Host Enhancement Scheme for TCP over Satellite IP Networks
}

\author{
Kui-Fai Leung and Kwan L. Yeung \\ Department of Electrical and Electronic Engineering \\ The University of Hong Kong \\ Hong Kong, PRC. \\ Tel: (852) 2857-8493 Fax: (852) 2559-8738
}

\begin{abstract}
A new transport layer protocol called TCPSwift is proposed for enhancing the TCP performance over satellite IP networks. TCP-Swift replaces the conventional TCP slow start and fast recovery algorithms by speedy start and speedy recovery. With speedy start, a TCP-Swift sender opens up its congestion window in only two round trip times. This significantly shortens the time needed in probing the network for equilibrium state. With speedy recovery, we can infer the cause of a packet loss by observing the $A C K$ stream received at the sender. If the loss is due to wireless transmission error, the sender's congestion window can be re-opened up more aggressively to fully utilize the available satellite link bandwidth. We show that TCP-Swift outperforms existing TCP schemes by simulations.
\end{abstract}

\section{Introduction}

The market need for Internet access via broadband satellite networks is increasing. Although TCP has been designed, improved and tuned to work efficiently in the wireline network, extending it to satellite networks is problematic. This is mainly due to the following satellite link characteristics.

Large bandwidth-delay product. TCP spends a long time in its slow start phase because of long latency of the satellite link. Current TCP applications, like HTTP, consist of many transfers of small files. Very likely such transfers will be completed within the slow start phase. In other words, TCP may not be able to fully utilize the available bandwidth of the network.

Transmission errors in satellite links. TCP interprets packet loss as a signal of network congestion, which then triggers the congestion control mechanism to reduce the transmission window size. Such interpretation is no longer valid when applied to satellite links. Due to factors like atmospheric conditions, RF interference, weak signal and so on, satellite links usually experience high bit error rate (BER).

Channel asymmetric. In many satellite systems, the bandwidths on the forward and backward channels are asymmetric. This is mainly due to cost limitation, increased transmitter power, and antenna size requirements for high bandwidth transmission. Using slower reverse channels can make more cost effective designs and save the scarce satellite bandwidth.

Many studies [1-2,4,6-8] have been carried out to enhance the performance of TCP over satellite IP networks. Recently an efficient scheme called TCPPeach [4] was designed. It adopts two new algorithms, sudden start and rapid recovery. Dummy segments are used in these algorithms for fast probing the availability of the network resources.

In this paper, a new transport layer protocol called TCP-Swift is proposed based on TCP-Peach. TCPSwift replaces the conventional TCP slow start and fast recovery algorithms by speedy start and speedy recovery. With speedy start, a TCP-Swift sender opens up its congestion window size in only two round trip times (RTTs). With speedy recovery, we can infer the cause of a packet loss by observing the ACK stream received at the sender. If a packet loss is due to wireless transmission error, the sender's congestion window can be re-opened up more aggressively to fully utilize the available bandwidth. In the next section, we first sunmarize our two major observations on TCP-Peach. Then TCP-Swift is proposed in Section III based on these observations. The performance of TCP-Swift is evaluated in Section IV. Finally we conclude the paper in Section V.

\section{Observations on TCP-Peach}

\section{A. Using Dummy Segment}

The dummy segments used in TCP-Peach [4] are lowpriority segments generated by the sender as a copy of the last transmitted data segment. Efforts on transmitting dummy segments into the network can be treated as the network overhead since they do not carry any new information to the receiver (assuming the last transmitted data segment is correctly received). Dummy segments may be transmitted through the network but dropped in subsequent routers along the transmission path. Hence dummy segments may cause network inefficiency. 


\section{B. Window Expanding Period (WEP)}

The information that conveyed by ACKs for data segments after rapid recovery [4] is not fully utilized. TCP-Peach sends a number of dummy segments to probe the availability of the network resources and expand its congestion window size according to the receipt of ACK for these dummy segments. Fig. 1 shows the corresponding window expanding period (WEP). WEP lasts for one RTT which is the period from the time the sender exiting/quitting the rapid recovery algorithm to receiving the ACK of the last data sègment sent in the rapid recovery [4].

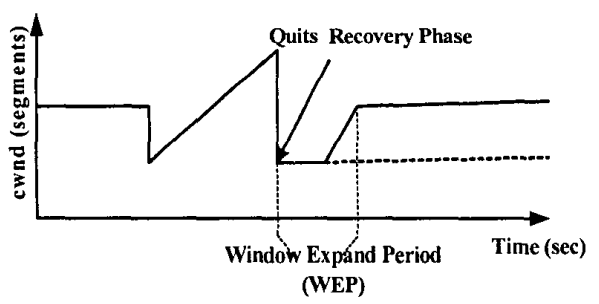

Fig. 1. Window Expanding Period

In rapid recovery, $c w n d_{\delta} / 2$ data segments and $c w n d_{0}$ dummy segments are injected into the network. During the WEP, the TCP sender will receive dummy ACKs (i.e. ACKs for dummy segments) and data ACKs (i.e. ACKs for regular data segments) respectively. TCPPeach starts increasing its congestion window upon receiving the $\left(c w n d_{0} / 2+I\right)$-th dummy ACK. Similar to the implementation of TCP-Reno, TCP-Peach enters the congestion avoidance phase after exiting the rapid recovery. Thus the congestion window is increased by one for each RTT during the congestion avoidance phase.

TCP is designed to adapt its transmission rate to bandwidth along the sender-to-receiver's transmission path. The return of data ACKs (for those data segments transmitted during rapid recovery) during WEP does indicate to the sender that there are still resources available in the network. As such, the congestion window should be further expanded for each data ACK received during WEP.

\section{Our Approach: TCP-Swift}

\section{A. Outstanding Segment \& Speedy Start}

To increase the network bandwidth utilization, we replace the dummy segments by outstanding segments for probing the available network resources. Dummy segment is a copy of the last transmitted data segment, whereas outstanding segments are chosen randomly from the set of packets/segments that have been sen but not-yet-acknowledged. So they serve as a backup in case the earlier sent packets are lost. This gives some extra loss repairing capability as compared with the dummy segment approach. Please note that the outstanding segment concept and the NIL segments in [9] share the same idea.

The priority implementation of outstanding segments can be supported in IP layer by the Type of Service (TOS) option in IPv4. Some recent commercial routers, like Cisco 7000 series and 12000 series emerge to support IP TOS.

The speedy start algorithm is summarized in Fig. 2 for replacing the original sudden start of TCP-Peach.

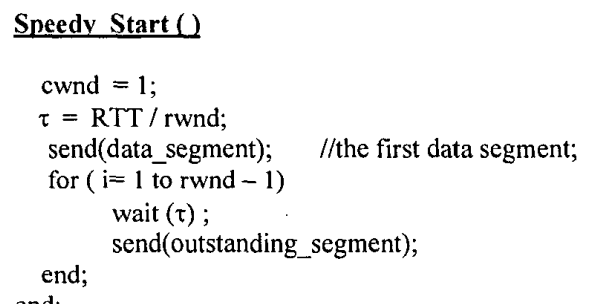

Fig. 2. Speedy start algorithm

\section{B. Congestion Window Increment Function \& Speedy Recovery}

To improve the efficiency of congestion window increment in WEP for a large bandwidth-delay product transmission path (like satellite links). We propose that TCP sender should be aggressive in increasing its transmission rate in order to fill up the available transmission pipe.

In our TCP-Swift, the resulting algorithm is called speedy recovery, as detailed in Fig. 3. (The definitions of variables used are described in Table 1.) A congestion window increment function is added to the speedy recovery. The operation of this function is described as follows. When a sender exits from the rapid recovery in. Fig. 1 (i.e. also the start time of WEP), the sequence number of the last data segment sent is recorded in a variable swift wep recv. During WEP, in case of the arrival of dummy ACKs, the sender behaves like TCP-Peach. For each data ACK arrived, the sender increases its congestion window by one until the ACK corresponding to the value of swift_wep_recv is received. Therefore, the congestion window size opens up more quickly and aggressively than rapid recovery of TCP-Peach. In short, speedy recovery differs from TCP-Peach's rapid recovery algorithm in two ways:

- Outstanding Segments are used instead of dummy segments. This replacement helps receiver to recover extra segment losses.

- Congestion Window Increment Function is added during the WEP. The sender's window keeps on growing during the WEP so that TCP-Swift is more aggressive to increase its transmission rate after an event of segment loss. 


\section{Speedy Recovery()}

aosn = cwnd; $w d s n=c w n d ~ / 2 ;$

apsn $=0$;

pipe $=$ cwnd - ndupacks +1 ;

$\mathrm{END}=0$;

while $(\mathrm{END}=0)$

if (ACK ARRIVE)

if (Duplicate ACK)

pipe $=$ pipe -1 ;

update scoreboard;

cwnd $=$ cwnd +1

if (ACK_for_outstanding_segment)

if (wdsn $>0$ )

wdsn $=w d s n-1$ end;

end

end;

else if (Partial ACK)

cwnd $=$ cwnd +1 ;

pipe $=$ pipe - amountacked;

update HighACK;

update scoreboard;

else if $(A C K<$ swift_wep_recv)

cwnd $=$ cwnd $+\overline{1}$;

//congestion window increment; end;

if (Recover ACK)

update HighAck;

clear scoreboard;

swift_wep_recv = $\mathrm{tSeq}$;

End $=1$;

end;

adps = cwnd - pipe;

nss $=\min (\max b u r s t$, adsn $)$;

if ( $\mathrm{nss}>0$ )

send nss missing packets and/or new packets;

pipe $=$ pipe + nss

else if (adps $>0$ )

send outstanding segment;

send outstanding segment;

$\operatorname{aos} n=\operatorname{aos} n-2$

end;

end; end

Fig. 3. Speedy recovery algorithm.

\section{Performance Evaluation}

We evaluate the performance of TCP-Swift by simulations using ns version 2.1b7a [10]. For comparison, TCP NewReno, TCP NewReno with SACK option [3], TCP-Peach, and TCP-Peachplus [9] are also implemented. We assume that a large file is transmitted between each sender-receiver pair using FTP protocol. Other related simulation parameters are summarized in Table 2.

\begin{tabular}{|c|c|}
\hline Variable & Definition \\
\hline Aosn & $\begin{array}{l}\text { number of outstanding data segment can } \\
\text { be sent during Speedy Recovery }\end{array}$ \\
\hline$W d s n$ & $\begin{array}{l}\text { preventing sender to increase its } \\
\text { congestion window size for } c w n d_{1} / 2 \\
\text { ACKs for outstanding segments }\end{array}$ \\
\hline Adps & number of segments allowed to be sent \\
\hline Nss & number of segments have been sent \\
\hline Pipe & $\begin{array}{l}\text { number outstanding of segments in the } \\
\text { network during speedy recovery }\end{array}$ \\
\hline HighACK & $\begin{array}{l}\text { sequence number of the highest } \\
\text { cumulative } A C K \text { received }\end{array}$ \\
\hline Partial $A C K$ & $\begin{array}{l}\text { an } A C K \text { that increases the HighACK } \\
\text { value but not acknowledging all sent } \\
\text { packets }\end{array}$ \\
\hline$T_{-}$Seqno_ & $\begin{array}{l}\text { sequence number of the current segment } \\
\text { sent }\end{array}$ \\
\hline Ndupacks & $\begin{array}{l}\text { number of duplicated ACKs to trigger } \\
\text { speedy recovery (a value of } 3 \text { is used). }\end{array}$ \\
\hline Amountacked & $\begin{array}{l}\text { number of segments have been } \\
\text { acknowledged }\end{array}$ \\
\hline$s w i f t$ & $\begin{array}{l}\text { sequence number of the last segment } \\
\text { sent when exits from rapid recovery. }\end{array}$ \\
\hline
\end{tabular}

Table 1 Variable definitions in speedy recovery algorithm of TCP-Swift.

\begin{tabular}{l|c|c}
\hline \multicolumn{1}{|c|}{ Parameters } & Symbols & Values \\
\hline $\begin{array}{l}\text { Number of senders/Receivers } \\
\begin{array}{l}\text { Buffer size at ground stations } \\
\text { A \& B }\end{array}\end{array}$ & $\mathrm{N}$ & 20 \\
\hline Receiver's window size & rwnd & 64 segments \\
\hline Satellite link capacity & $\mathrm{C}$ & $10 \mathrm{Mbps}$ \\
\hline $\begin{array}{l}\text { Satellite link packet loss rate } \\
\text { Round trip time of satellite } \\
\text { link }\end{array}$ & $\mathrm{P}_{\text {loss }}$ & $10^{-2} \sim 10^{-5}$ \\
\hline $\begin{array}{l}\text { Initial TCP Slow Start } \\
\text { threshold }\end{array}$ & ssthresh & $550 \mathrm{~ms}$ \\
\hline TCP segment size & Packet_size & 1000 bytes \\
\hline ACK size & ACK_size & 40 bytes \\
\hline
\end{tabular}

Table 2 Simulation Parameters

\section{A. Goodput Performance}

Our first set of simulations is based on the network topology shown in Fig. 4. The system consists of $\mathrm{N}$ sender-receiver pairs. The senders and receivers are connected through satellite channel by ground stations $\mathrm{A}$ and $\mathrm{B}$. In this simulation we consider a GEO satellite system where the round trip time of the satellite link is $550 \mathrm{~ms}$, and a bandwidth of $10 \mathrm{Mbps}$ is assumed. All terrestrial links are characterized by $1 \mathrm{~ms}$ propagation delay and $10 \mathrm{Mbps}$ bandwidth. The router buffer sizes at ground stations $\mathrm{A}$ and $\mathrm{B}$ are set to 50 and managed by droptail queuing mechanism.

Fig. 5 shows the goodput performance of TCP-Swift against the packet error rate. We can see that TCP-Swift gives the highest goodput performance. This accredits to the speedy start and seedy recovery algorithms adopted by TCP-Swift. We can also see that TCP- 
NewReno with SACK option outperforms the general TCP-NewReno. This shows that SACK option can overcome the problem of multiple segment losses within a transmission window.

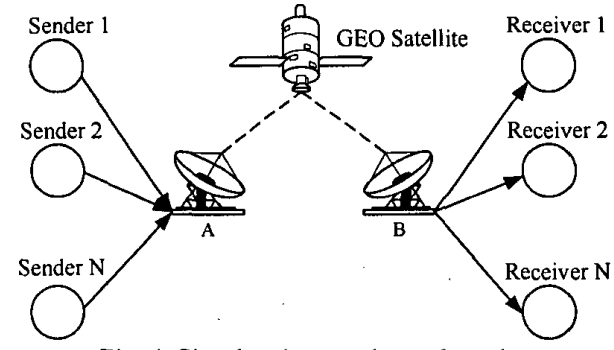

Fig. 4. Simulated network topology 1.

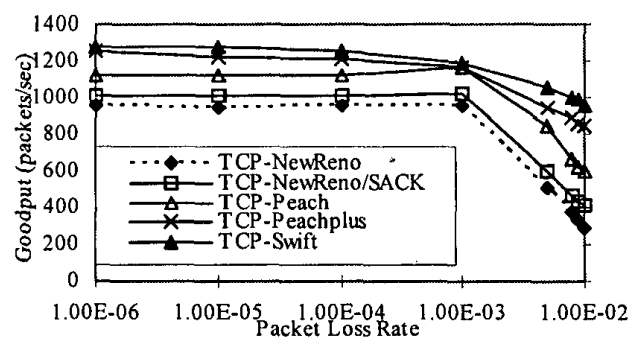

Fig. 5. Goodput performance comparison.

\section{B. Fairness Performance}

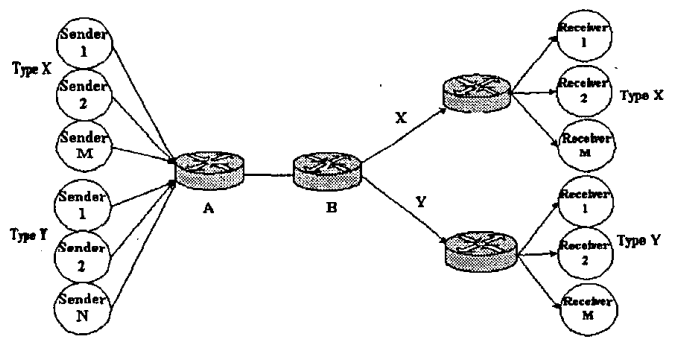

Fig. 6. Simulated network topology 2.

We study the case of heterogeneous scenario by considering the network topology shown in Fig. 6 . There are $\mathrm{M}$ connections of type $\mathrm{X}$ and $\mathrm{N}$ connection of type Y. All connections pass through the same bottleneck link that connects routers $\mathrm{A}$ and $\mathrm{B}$. The bottleneck link capacity is $10 \mathrm{Mbps}$. We assume type $\mathrm{X}$ connections pass through a GEO satellite link with round trip time $R T T_{X}=550 \mathrm{~ms}$, and packet loss probability $P_{\text {Loss }}$ varied from $10^{-6}$ to $10^{-2}$. On the other hand, type $Y$ connections pass through a terrestrial link, where no losses occur due to link error, i.e. $P_{\text {Loss } Y}=0$ and the round trip time $R T T_{Y}$ for the terrestrial link is set to $200 \mathrm{~ms}$ [4]. In our simulations, the number of connections $\mathrm{X}$ and connections $\mathrm{Y}$ are set to be the same
$(M=N=5)$, and the bottleneck router buffer size is set to 50 and $r w n d=64$ segments.

The fairness performance is measured by the ratio between the average goodput $\left(r_{X}\right)$ of type X's connections and the average goodput $\left(r_{Y}\right)$ of type Y's connections. So $r_{X} / r_{Y}=1$ gives the ideal fairness performance. In Fig. 7 , we show that the fairness performance in the heterogeneous scenario. The legend "NewReno \& Swift" means that connections of type X use TCP-Swift passing through satellite link and connections of type $\mathrm{Y}$ use TCP-NewReno passing through terrestrial link.

From Fig. 7, we can see that "NewReno \& Swift" gives the best fairness performance as compared to BewReno \& Peach" and "NewReno \& NewReno". This is due to TCP-Swift's aggressiveness in maintaining the high transmission rate. So TCP-Swift can acquire enough network resource when competing with TCPNewReno.

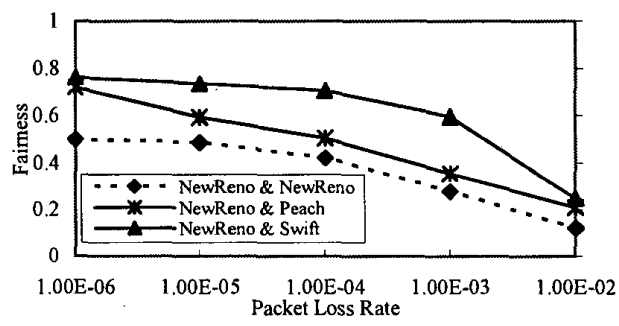

Fig. 7. Fairness performance comparison.

\section{Conclusions}

In this paper, a new transport layer protocol called TCP-Swift was proposed for enhancing the TCP performance over satellite IP networks. TCP-Swift replaces the conventional TCP slow start and fast recovery algorithms by speedy start and speedy recovery. With speedy start, a TCP-Swift sender opens up its congestion window in only two round trip times. This significantly shortens the time needed in probing the network for equilibrium state. With speedy recovery, we can infer if a packet loss is due to wireless transmission errors by observing the ACK stream arrived at the sender. The sender's congestion window can be re-opened up more aggressively if the loss is due to transmission error.

\section{Acknowledgements}

This work was supported by Competitive Earmarked Research Grant HKU 1180/00E.

\section{References:}

[1] C. Metz, "TCP over satellite... The final frontier," IEEE Internet Computing, pp. 76-80, Jan./Feb. 1999. 
[2] M. Allman et al., "Ongoing TCP research related to satellites," RFC 2760, Feb. 2000.

[3] M. Mathis, J. Mahdavi, S. Floyd and A. Romanow, "TCP Selective Acknowledgment Options," RFC 2018, Oct. 1996.

[4] I. F. Akyildiz, G. Morabito, S. Palazzo, "TCP Peach: A New Congestion Control Scheme for Satellite IP Networks," IEEE/ACM Transactions on Networking, 307-321. Jun. 2001.

[5] V. N. Padmanabhan and R. H. Katz, "TCP Fast Start: A Technique for Speeding Up Web Transfers," Proc. IEEE Globecom'98 Internet Mini-Conference, Sydney, Australia, November 1998.

[6] C. Partridge and T. J. Shepard, "TCP/IP performance over satellite links," IEEE Network Mag., pp. 44-49, Sept./Oct. 1997.

[7] T. R. Henderson and R. H. Katz, "Transport protocols for Internet-compatible satellite networks," IEEE J. Select. Areas Commun,, vol. 17, pp. 326-344, Feb. 1999.

[8] M. Allman, "On the generation and use of TCP acknowledgments," ACM Computer Commun. Review, vol. 28, Oct. 1998.

[9] I.F. Akyildiz, X. Zhang and J. Fang, "TCP-Peach+: Enhancement of TCP-Peach for Satellite IP Networks," IEEE Communications Letters, vol. 6 Issue: 7, pp. $303-305$, Jul. 2002

[10] S. McCanne and S. Floyd. Ns-LBNL Network Simulator. URL: http://www-nrg.ee.lbl.gov/ns/ 